\title{
Diferencial de mortalidade em homens e mulheres em localidade da região Sudeste, Brasil - 1960, 1970 e 1980.
}

\author{
The sex differential in mortality a city of Southeastern Brazil - 1960, 1970, 1980.
}

\author{
Dóra Chor*, Milena Piraccini Duchiade*, Angela Maria Fernandes Jourdan*
}

\begin{abstract}
CHOR, D. et al. Diferencial de mortalidade em homens e mulheres em localidade da regiāo Sudeste, Brasil - 1960, 1970, 1980. Rev. Saúde Públ., S. Paulo, 26: 246-55, 1992. Foram comparadas as taxas de mortalidade masculinas e femininas no Município do Rio de Janeiro (Brasil), em 1960, 1970 e 1980 , buscando analisar os diferentes riscos a que homens e mulheres são submetidos, em cada grupo etário. Os diferenciais de mortalidade por sexo e causa foram estudados através da Razäo de Sobremortalidade Masculina, das diferenças relativas e absolutas entre taxas e de taxas padronizadas. Além disso, foi desenvolvida uma análise dos diferenciais por grupos selecionados de causas para o ano de 1980. As taxas de mortalidade masculinas foram maiores do que as femininas em todas as faixas etárias, nos três anos estudados, com aumento da Razão de Sobremortalidade Masculina entre 15 e 34 anos, no periodo considerado. O excesso de mortes masculinas foi causado sobretudo pela elevação dos óbitos por causas violentas em homens jovens, o que retrata uma realidade dramática, cuja possibilidade de transformação através de medidas "técnicas" é escassa. Em relação aos óbitos por outras causas, os determinantes biológicos e os diferentes riscos a que são submetidos homens e inulheres devem ser considerados, buscando-se melhor compreensão da realidade.
\end{abstract}

Descritores: Mortalidade diferencial. Fatores sexuais. Coeficiente de mortalidade. Análise de risco.

\section{Introdução}

A identificação de características individuais, como sexo e idade, faz parte do modelo clássico de análise da distribuição de agravos à saúde que ocorrem em populações desde os primeiros estudos que utilizaram dados de mortalidade. A doença é observada, então,de acordo com variáveis ditas intrínsecas, inerentes à pessoa, e variáveis extrínsecas, como o tempo e o lugar onde as doenças acontecem (Lilienfeld, e Lilienfeld $\left.{ }^{16}, 1980\right)$. De acordo com a presença ou ausência destas caracteristicas, os indivíduos são agrupados, buscando-se identificar semelhanças e diferenças entre os que adoecem ou não, entre aqueles que morrem ou sobrevivem. Desta forma, a epidemiologia vem contribuindo no esclarecimento das causas de doença e morte dos diversos grupos populacionais.

Ser homem ou ser mulher é um dos determinantes mais importantes da inserção dos indivíduos no mundo. Esta inserção é construída ao longo da vida, através de valores sociais e culturais, muitos deles diferentes segundo a classe

\footnotetext{
* Departamento de Epidemiologia e Métodos Quantitativos em Saúde da Escola Nacional de Saúde Pública (FIOCRUZ) - Rio de Janeiro, RJ - Brasil.

Separatas/Reprints: D. Chor - Rua Leopoldo Bulhões, 1480 $8^{2}$ andar - 2104 1-210 - Rio de Janeiro, RJ - Brasil.
}

social. A categoria classe social, no entanto, não explica de forma suficiente os modos de vida distintos de homens e mulheres. Castro 3 (1991), adverte sobre os riscos de duas posturas que empobrecem a discussão sobre as diferenças entre os sexos. A primeira,"aponta a forma de organização da produção da sociedade como causa única destas diferenças". A segunda, identifica"a cultura ou a ideologia em abstrato, como planos por excelência para compreensão da situação da mulher". Compreendemos, por outro lado, que as diferenças de gênero (sexo construído culturalmente) ocorrem numa sociedade de classes, e estes dois componentes determinam modos de vida diferentes, afetando a forma e o momento em que homens e mulheres adoecem, e portanto, as condições que os levam à morte.

Nos paises desenvolvidos, e também no Brasil, é sabido que, atualmente, as mulheres vivem mais do que os homens. Indicando este fato, a Esperança de Vida ao Nascer (EVN) de mulheres, em paises como França, Canadá e Estados Unidos, em 1988, foi entre 7 e 8 anos maior do que a dos homens. Esta diferença situava-se entre 5 e 7 anos em 1965, o que demonstra o aumento da distância entre as EVN de homens e mulheres, nas últimas décadas, nesses países (Banco Mundial $\left.{ }^{1}, 1990\right)$.

Esta "vantagem feminina" é verificada em 
quase todo o mundo, com raras exceções. India, Paquistāo e Bangladesh apresentaram, em 1988, a mesma EVN para ambos os sexos, enquanto no Nepal e na China, os homens apresentaram apenas um ano a mais (Banco Mundial $\left.{ }^{1}, 1990\right)$. Altos indices de mortalidade materna, entre 340 e 830 por 100.000 nascidos vivos na década de 80 (UNICEF $^{25}$, 1991), nos quatro primeiros países citados, podem explicar parte desta situação. $\mathrm{Na}$ verdade, a mortalidade materna reflete o "status" da mulher naquelas sociedades, sua saúde e nutrição, sua educação e carga de trabalho. Outros fatores, como a diferente valorização social de meninos e meninas, devem contribuir para esta situação em países como a China, cuja mortalidade materna tem sido baixa, 44 por 100.000 nascidos vivos em 1980 (Banco Mundial $\left.^{1}, 1990\right)$ e a India, onde Basu ${ }^{2}$ (1989) aponta a busca diferenciada de cuidados de saúde segundo o sexo da criança. Na América Latina dos anos 50 eram verificados fenômenos semelhantes. Alguns países como Guatemala, Panamá e Jamaica exibiam taxas de mortalidade feminina superiores às taxas masculinas, entre 15 e 24 anos (Mortara ${ }^{18}$, 1962).

O Brasil, seguindo o padrão mundial, vem apresentando maiores niveis de EVN para as mulheres nos últimos 50 anos (Fig. 1). Não se observa, no entanto, o aumento da diferença apontado para os países desenvolvidos, já que a distância entre os sexos se manteve em torno de 5 anos. $\mathrm{Na}$ verdade, no último periodo, 1980 1988, observa-se um ganho maior para os homens (6 anos) do que para as mulheres $(4,6$ anos), tendendo portanto à diminuição da distância entre as respectivas EVN. Entre 1940 e 1988 , houve incremento praticamente igual na EVN de ambos os sexos, cerca de 24 anos. As-

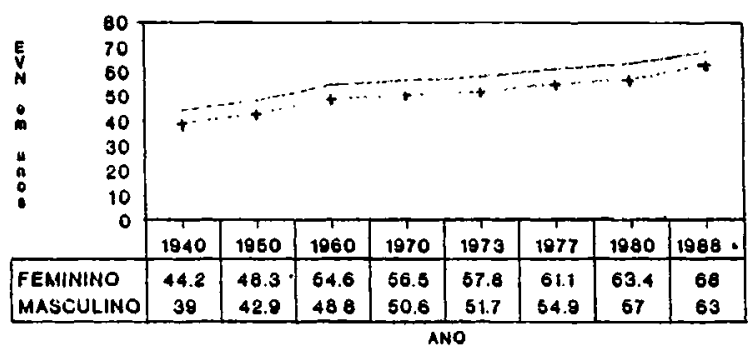

- FEMININO + MASCULINO

Figura 1. Esperança de vida ao nascer por sexo. Bra. sil $1940 / 1980$.

Fonte: IBGE ${ }^{10},(1984)$ Indicadores Sociais

- Banco Mundial", 1990. sim, a EVN para os homens, passou de 39 para 63 anos, e das mulheres, de 44,2 para 68 anos (IBGE, ${ }^{10}$ 1984; Banco Mundial ${ }^{1}, 1990$ ).

Buscando-se explicaçōes para o diferencial de mortalidade entre homens e mulheres, a ligação entre condições sociais e experiência biológica é provocante. Se, por um lado, é quase intuitiva a relação entre a magnitude e a causa do óbito com as diferentes inserções sociais, o que implica diferentes exposiçōes a "situações de risco" (Johnson $^{13}$, 1977; Gove e Hughes ${ }^{12}$, 1979; Enterline ${ }^{4}$, 1961), não é possível excluir a participação das diferenças biológicas do modelo explicativo.

A sobremortalidade masculina desde o período pré-natal e a infância (Vallin e Meslé26, 1988; Waldron ${ }^{28}, 1983$ ) aponta no sentido de uma "maior adequação biológica" das mulheres (Wingard ${ }^{29}, 1982$ ). No entanto, também nestes dois periodos de vida, há dificuldade em estabelecer limites precisos entre o que é conseqüência das condiçōes sociais e o que é efeito de diferenças biológicas. A razão de sexo (número de homens por 100 mulheres) ao nascer, pode ser indiretamente influenciada por fatores sociais, que atingem o feto no útero. Como os fetos masculinos têm maior risco de abortamento e natimortalidade, devido à maior incidência de alteraçōes genéticas, as populaçōes que apresentam maiores indices de abortamento espontâneos e natimortalidade apresentam número relativamente menor de nascidos vivos do sexo masculino. É reconhecida a determinação social da freqüência de abortamentos e natimortalidade, seja pela precária assistência pré-natal ou pela situação de saúde da mulher.

A imbricação de fatores biológicos e sociais se estende à mortalidade infantil que é maior, na maioria dos países, no sexo masculino. Alguns autores admitem que este padrão é biologicamente determinado (Wingard ${ }^{30}, 1984$ ). Por outro lado, diversos deteterminantes da mortalidade infantil, como assistência à saúde, condiçōes de moradia e outros, podem influenciar a razão de sexo dos óbitos neste período. Ferreira e Flores $^{5}$ (1987) observaram, no Município de São Paulo, que à medida que a mortalidade infantil se concentrou no período perinatal, onde $o$ risco de óbito masculino é relativamente maior do que nos períodos posteriores, a participação deste sexo, na mortalidade infantil, tornou-se progressivamente mais importante. Assim, naquela cidade, em 1940, a mortalidade de meninos menores de um ano era cerca de $10 \%$ mais elevada do que de meninas. Já no final da década de 70 , a diferença passou a ser de $25 \%$.

Numa espécie de mecanismo compensató- 
rio, apesar da maior mortalidade de fetos masculinos, a razão de masculinidade é favorável aos homens, ao nascer. Esta vantagem, no entanto, vai desaparecendo com o correr dos anos, devido à sobremortalidade masculina, particularmente evidente entre 15 e 35 anos. Assim, na população geral há excesso de mulheres, que representavam $50,3 \%$ da população brasileira total, em 1980 (IBGE9, 1983 ). Claro está que, nas idades avançadas, a sobremortalidade masculina tende a desaparecer, já que a morte é inevitável para ambos os sexos.

As causas específicas dos óbitos de homens e mulheres refletem diferenças importantes entre os modos de viver e morrer. Comparando-se às mulheres, em 1980, os homens brasileiros maiores de 15 anos, tiveram 10 vezes mais chance de morrer por homicídios, 3,5 vezes mais por acidentes de transporte, 3,5 vezes mais por cirrose, 3 vezes mais de câncer de pulmão, e finalmente, quase 2 vezes mais de infarto agudo do miocárdio. por outro lado, dentre as causas de óbito mais freqüentes no país, a mortalidade feminina foi superior à masculina somente por diabetes mellitus, com uma diferença relativa a 1,5 (Ministério da Saúde ${ }^{17}, 1983$ ).

O objetivo do presente estudo é a descrição dos diferenciais de mortalidade entre os sexos, em três momentos, $1960,1970,1980$, no Município do Rio de Janeiro, com ênfase na discussão das causas específicas, no último ano.

\section{Metodologia}

O Município do Rio de Janeiro caracterizase por uma população eminentemente urbana, com idade mediana elevada, tendendo ao envelhecimento, devido à queda da fecundidade desde a década de 60. A força de trabalho concentra-se quase que exclusivamente nos setores secundário e terciário, sendo que desde 1970, menos de $1 \%$ da população economicamente ativa trabalhava no setor primário. A densidade demográfica é alta - 4.343,31 habitantes $/ \mathrm{km}^{2}$ - em 1980, embora apresente-se de modo diferenciado segundo as várias Regiões Administrativas que compõem a cidade. Os indicadores de saneamento revelam disparidades entre o núcleo e a periferia imediata, em relação às zonas mais distantes. Mesmo assim, em 1980, apenas 2 das 24 Regiões Administrativas apresentavam menos de $80 \%$ dos domicílios ligados à rede geral de água (IBGE 9 , 1983). A cidade passou por grandes transformações nas últimas décadas, e concentra enormes contrastes sociais, apresentando perfil de mortalidade típico de um grande centro urbano da América Latina.

As informações sobre os óbitos ocorridos no Município do Rio de Janeiro, em 1980, foram obtidas do Ministério da Saúde ${ }^{17}$, através de publicação específica e de fita para computador de grande porte, onde se encontram todos os dados da Declaração de Óbito. Os óbitos cortespondentes a 1960 e 1970 foram obtidos da publicação da Fundação Instituto de Desenvolvimento Econômico e Social do Rio de Janeiroll (1977). Em relação à população, foram utilizados os dados provenientes dos Censos realizados em 1960, 1970 e 1980 (IBGE ${ }^{7,8,9}, 1960,1970$, 1980).

O diferencial entre as taxas de mortalidade, específicas por sexo, pode ser medido de duas formas, através da razão ou da diferença absoluta entre os coeficientes. Cada uma delas fornece informação distinta. Enquanto a razão entre taxas, aqui chamada Razão de Sobremortalidade Masculina (RSM), informa sobre a diferença relativa entre as taxas específicas por sexo, a diferença absoluta mede a magnitude do diferencial. Por outro lado, o comportamento da diferença absoluta é influenciado tanto pela evolução do diferencial da taxa de mortalidade entre os sexos quanto pela evolução do tisco geral de mortalidade, enquanto a razão informa melhor sobre o comportamento específico da relação entre as taxas de cada sexo.

No caso, por exemplo, da taxa de mortalidade dos homens de um determinado grupo etário ou população ser da ordem de $20 / 100.000$ e das mulheres, $10 / 100.000$, num primeiro momento, e num segundo, 40/100.000 nos homens e 20/100.000 nas mulheres, o diferencial entre as taxas específicas de mortalidade, medido através da razão, não se alterou, informando que a relação entre as taxas permaneceu igual a 2. Já a diferença absoluta tornou-se duas vezes maior, por ser determinada também pela magnitude de cada taxa, além da relação entre elas. Assim, enquanto a razão entre taxas informa melhor sobre mudanças na relação entre as taxas especificas por sexo, ao longo do tempo, a diferença absoluta é útil na estimativa da contribuição de cada causa de óbito no diferencial geral, entre homens e mulheres. No caso do presente estudo, as duas formas de medida foram utilizadas, já que fornecem informaçōes diferentes e complementares.

Utilizando a razão ou a diferença entre as taxas de mortalidade, as distribuições etárias distintas de homens e mulheres devem ser levadas em consideração. Uma vez que as mulheres vivem mais tempo do que os homens, a população 
feminina, considerada em conjunto, é mais velha do que a população masculina. Desta forma, as taxas brutas de mortalidade (ou taxas de mortalidade geral) podem ser maiores, entre as mulheres, pelo simples fato de existir mais mulheres idosas, e não por estarem mais expostas a situações de risco. Aliás, o fato de viverem mais, indicado pela maior EVN das mulheres, revela exposições de qualidade diferente, ao longo da vida. Por este motivo, ao compararmos a mortalidade específica por sexo, de toda a população, utilizamos coeficientes padronizados ou ajustados por idade. Além disso, a padronização de coeficientes pela idade foi utilizada para compararmos as taxas de mortalidade específicas por sexo em 1960, 1970 e 1980, período em que a população do Município, en conjunto, envelheceu. Com os indicadores ajustados pela idade, as diferenças encontradas entre as taxas de mortalidade, masculina e feminina, não podem mais ser explicadas pelas distribuições etárias distintas, mas por outros conjuntos de fatores.

Apesar da simplicidade de execução e de ser um dos métodos mais tradicionais de ajustamento de dados, a padronização não pode ser utilizada em qualquer situação. Isto, porque, como qualquer medida que pretende "resumir" informações, pode mascarar a situação real, refletida nas taxas específicas por idade. Autores como Fleiss ${ }^{6},(1981)$ e Kahn ${ }^{14}$, (1989), entre outros, chamam atenção para o fato de que nenhum índice geral, bruto ou ajustado, substitui satisfatoriamente a observação e análise das taxas de cada grupo etário. A perda de informação pode ser de maior ou menor monta, dependendo de cada situação. Assim, diante de duas populações em que as taxas específicas por idade são distintas para diferentes grupos etários (por exemplo, uma população com mortalidade precoce elevada e outra com mortalidade tardia elevada) a taxa de mortalidade geral, padronizada, não será capaz de retratar fielmente esta realidade. Nestes casos, seriam necessárias duas taxas ajustadas, dividindo-se as populações nos grupos etários em que a magnitude da taxa específica por idade torna-se maior na segunda população. Em outros casos, onde a variação das taxas especificas por idade é ainda maior, o coeficiente padronizado torna-se completamente sem significado.

No caso do presente estudo, as taxas de mortalidade da população feminina apresentaram-se consistentemente maiores do que as masculinas, em todos os grupos etários, e em cada ano. Assim sendo, a escolha da população padrão, outro assunto bastante discutido pelos au- tores, tornou-se menos problemática. Optou-se, então, pela população geral do município, estudado, em 1970, por ser a intermediária, para o cálculo da taxa de mortalidade geral. Ao compararmos a mortalidade por sexo, segundo causas de óbito em 1980, observamos situaçōes semelhantes, com as taxas masculinas sempre superiores às femininas, exceto no caso do diabetes. Escolheu-se, então, a própria população de mulheres, residentes no Rio de Janeiro, em 1980, como padrão, para o estudo dos diferenciais de mortalidade por causa.

A disponibilidade dos dados publicados referentes a 1960 e 1970 limitou de várias formas a análise realizada. Assim, por exemplo, a composição dos grupos etários comparados foi determinada pela divisão apresentada naqueles anos. A análise da mortalidade segundo causa do óbito, idade e sexo, em 1960, não constava da publicação utilizada. Por outro lado, nos dados relativos a 1970, só foi possível analisar os óbitos segundo grandes grupos de causa, já que não há, na publicação consultada, informações mais desagregadas. Por este motivo, não pôde ser comparada a evolução da Razão de Sobremortalidade Masculina por Homicídios, por exemplo, em 1970 e 1980.

Embora tenham sido utilizadas duas revisões diferentes da Classificação Internacional de Doenças (CID), a oitava ${ }^{19}$, para os óbitos que ocorreram em 1970,e a nona ${ }^{20}$, para 1980 , não houve mudanças nas regras de classificação dos grandes grupos comparados, evitando problemas quanto a critérios diferentes de tabulação dos óbitos. Foram analisados os seguintes grupos ou doenças: Tuberculose Pulmonar (CID 011); Pneumonia (CID 480-486); Causas Externas (CID 800-999); Doenças do Coração (englobando Febre Reumática - CID 390-392; Doenças Reumáticas do Coração - CID 393-398; Doença Hipertensiva - CID 400-404 na 8a Revisão e 401405 na 9 Revisão; Doenças Isquêmicas do Coração - CID 410-414; Outras Formas de Doença do Coração - CID 420-429 na 8 a Revisão; Doença da Circulação Pulmonar - CID 415-417 e Outras Formas de Doença do Coração - CID 420429 na 9ª Revisão; Doença Cerebrovascular (CID 430-438); Cirrose Hepática (8ª Revisão) e Doenças Crônicas do Fígado e Cirrose (9a Revisão) CID 571; Neoplasmas Malignos - CID 140-209 (8 Revisão) e CID 140-208 (9ª Revisão); Infarto Agudo do Miocárdio (CID 410); Tumores do Aparelho Respiratório (CID 162); Acidentes de Transporte (E800-E848); Homicídio (E960E969); Suicídio (CID E950-E959).

Outra limitação dos dados de 1960 e 1970 
refere-se à ausência de informação detalhada sobre os óbitos que ocorreram no Rio de Janeiro, embora não correspondessem a residentes do Município. Os óbitos passaram a ser registrados segundo o local de residência somente a partir de 1976. A única informação disponível referiase ao total de óbitos "invasores", 2.091 (6\% do total), em 1960 , e $3.328(8,6 \%$ do total) em 1970 , sem distribuí-los por idade e sexo. Não foi possível, portanto, descontar estes óbitos da mortalidade especifica por sexo e idade, implicando na superestimação das taxas de mortalidade de 1960 e 1970 , em relação à taxa de 1980 . Apesar disto, não houve maior comprometimento da análise, já que o objetivo principal do estudo não foi o de comparar a magnitude das taxas, ao longo do tempo, mas o de comparar o diferencial entre homens e mulheres. Já que não há motivos para acreditar que a "invasão" tenha ocorrido preferencialmente em um dos sexos ou em determinados grupos etários, mas de maneira mais ou menos uniforme em todos os subgrupos da população, pode-se supor que os diferenciais estimados entre taxas, objeto principal do presente estudo, estejam próximos daqueles que seriam obtidos caso a correção fosse realizada.

\section{Resultados}

As informações sobre a mortalidade específica por sexo e idade, bem como as taxas gerais, brutas e ajustadas por idade, encontram-se condensadas na Tabela 1 , relativas a 1960 ; na Tabela 2, a 1970 e na Tabela 3, a 1980.

A observação da diferença absoluta entre as taxas brutas de mortalidade, de homens e mulheres, torna evidente a sobremortalidade masculina, quando todas as causas de óbito são consideradas em conjunto. Em 1960 morteram 326 homens a mais do que mulheres, para cada 100.000 pessoas. Esta diferença diminui nos outros dois anos estudados, passando a $295 \mathrm{em}$ 1970, e 260 em 1980, o que pode ser explicado pela influĉncia da diminuição das taxas de mortalidade especificas por sexo, neste tipo de medida (vide metodologia).

A taxa bruta de mortalidade apresentou redução de cerca de $10 \%$ entre 1960 e 1970 , e também entre 1970 e 1980, tanto nos homens como nas mulheres. No entanto, à medida que diminuiu a mortalidade geral, houve aumento importante do excesso de mortalidade masculina em relação à feminina. Assim, a razão entre as taxas padronizadas pela idade, específicas por sexo Razão de Sobremortalidade Maculina (RSM) -
Tabela 1 - Taxas $\theta$ diferenciais de mortalidade por sexo e idade - Município do Rio de Janeiro - RJ, 1960.

\begin{tabular}{|c|c|c|c|c|}
\hline \multirow[b]{2}{*}{ Idade } & \multicolumn{2}{|c|}{ Taxas por 100.000} & \multirow{2}{*}{$\begin{array}{r}\text { Razāo } \\
\text { H/M }\end{array}$} & \multirow{2}{*}{$\begin{array}{r}\text { Diferença } \\
H / M\end{array}$} \\
\hline & $\mathrm{H}$ & $M$ & & \\
\hline 0.4 & $2.521,01$ & $2.221,04$ & 1,14 & 300 \\
\hline 5.14 & 131,05 & 93,28 & 1,40 & 38 \\
\hline 15.24 & 296,53 & 195,65 & 1,52 & 101 \\
\hline $25-34$ & 564,32 & 304,81 & 1,85 & 260 \\
\hline 35.44 & 926,15 & 481,39 & 1,92 & 445 \\
\hline $45-54$ & $1.581,02$ & 841,22 & 1,88 & 740 \\
\hline 55.64 & $3.211,00$ & $1.667,63$ & 1,93 & 1.543 \\
\hline $65 e+$ & $7.878,58$ & $6.056,13$ & 1,30 & 1.822 \\
\hline \multicolumn{5}{|c|}{ Todas as idades } \\
\hline Brutas(a) & $1.200,01$ & 873,65 & 1,37 & 326 \\
\hline $\begin{array}{l}\text { Padroniza- } \\
\text { das(b) }\end{array}$ & $1.274,03$ & 860,80 & 1,48 & 413 \\
\hline
\end{tabular}

H. Homens

M- Mulheres

(a) Inclui 2.091 óbitos (6.2\%) "importados" (ver Metodologia)

(b) Taxas padronizadas pela população total do Município em 1970

Tabela 2 - Taxas e diferenciais de mortalidade por sexo e idade - Município do Rio de Janeiro - RJ, 1970.

\begin{tabular}{lrrrr}
\hline & \multicolumn{2}{c}{ Taxas por 100.000} & \multicolumn{2}{r}{ Razão } \\
ldade & \multicolumn{1}{c}{$H$} & \multicolumn{1}{c}{$M$} & \multicolumn{1}{c}{ Diferença } \\
\hline 0.4 & $1.883,84$ & $1.569,41$ & 1,20 & 314 \\
5.14 & 98,09 & 65,36 & 1,5 & 33 \\
$15-24$ & 254,99 & 125,46 & 2,03 & 130 \\
$25-34$ & 430,86 & 225,31 & 1,91 & 206 \\
35.44 & 768,47 & 387,41 & 1,98 & 381 \\
$45-54$ & $1.451,39$ & 741,37 & 1,96 & 710 \\
$55-64$ & $2.928,36$ & $1.576,74$ & 1,86 & 1.352 \\
65 e + & $7.994,91$ & $5.927,53$ & 1,35 & 2.067 \\
\hline Todas as idades & & & \\
Brutas(a) & $1.067,56$ & 772,57 & 1,38 & 295 \\
$\begin{array}{l}\text { Padroniza. } \\
\text { das(b) }\end{array}$ & $1.131,38$ & 732,27 & 1,55 & 399 \\
\hline
\end{tabular}

H. Homens

M. Mulheres

(a) Inclui 3.328 óbitos $(8,6 \%)$ "importados" (ver Metodologia)

(b) Taxas padronizadas pela populaçāo total do Municipio em 1970

que era $48 \%$ maior para os homens, em 1960 (razão $=1,48$ ), tomou-se progressivamente maior. Situou-se em 55\% em 1970 (razão = 1,55) e, em 1980, a mortalidade masculina foi $62 \%$ superior à feminina (razão $=1,62$ ). 
Tabela 3 - Taxas e diferenciais de mortalidade por sexo e idade - Município do Rio de Janeiro - RJ, 1980

\begin{tabular}{|c|c|c|c|c|}
\hline \multirow[b]{2}{*}{ Idade } & \multicolumn{2}{|c|}{ Taxas por 100.000} & \multirow{2}{*}{$\begin{array}{r}\text { Razão } \\
H / M \\
\end{array}$} & \multirow{2}{*}{$\begin{array}{r}\text { Diferença } \\
H / M\end{array}$} \\
\hline & $\mathrm{H}$ & $M$ & & \\
\hline 0.4 & $1.177,10$ & 954,92 & 1,23 & 222 \\
\hline 5.14 & 80,08 & 60,94 & 1,31 & 19 \\
\hline $15 \cdot 24$ & 278,61 & 79,46 & 3,51 & 199 \\
\hline 25.34 & 378,54 & 151,98 & 2,49 & 227 \\
\hline $35-44$ & 625,71 & 307,69 & 2,03 & 318 \\
\hline $45-54$ & $1.235,56$ & 609,75 & 2,03 & 626 \\
\hline $55-64$ & $2.453,22$ & $1.255,40$ & 1,95 & 1.198 \\
\hline $65 e+$ & $7.240,10$ & $5.444,62$ & 1,33 & 1.795 \\
\hline \multicolumn{5}{|c|}{ Todas as idades } \\
\hline Brutas & 958,32 & 697,86 & 1,37 & 260 \\
\hline $\begin{array}{l}\text { Padroniza- } \\
\text { das(a) }\end{array}$ & 950,29 & 585,35 & 1,62 & 365 \\
\hline
\end{tabular}

H. Homens

M. Mulheres

(a)Taxas padronizadas pela população total do Município em 1970

No entanto, o aumento deste diferencial não correu de maneira uniforme nos diversos grupos de idade (Fig. 2). Houve deslocamento dos maiores diferenciais para as faixas etárias mais jovens, ao longo do tempo. Enquanto as maiores razōes encontravam-se entre 25 e 64 anos, em 1960 , e entre 15 e 64 anos, em 1970, a sobremortalidade masculina mais acentuada ocorreu no grupo de 15 a 34 anos, especialmente entre 15 e 24 anos, em 1980. Neste último estrato, a mortalidade dos homens foi 3,5 vezes maior do que nas mulheres, em 1980, o que pode ser explicado pclo aumento da taxa de mortalidade masculina neste grupo, cerca de $9 \%$ entre 1970

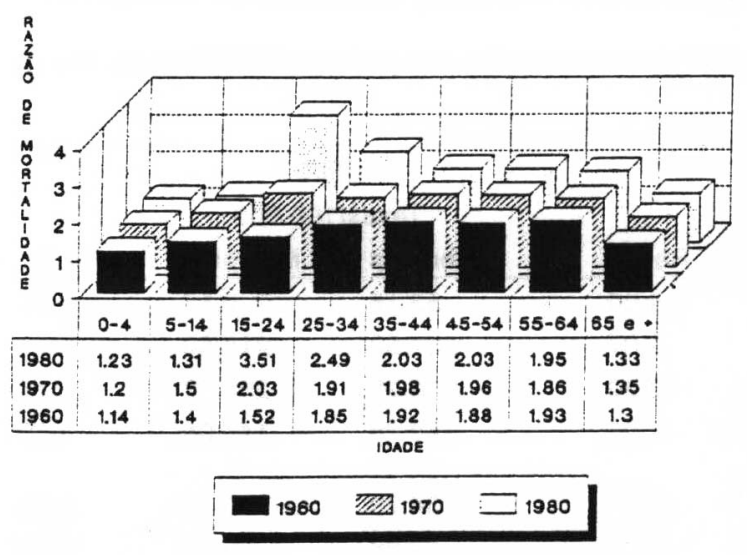

Figura 2. Razão da sobremortalidade masculina por idade. Rio de Janeiro, 1960, 1970, 1980. e 1980 , passando de $254,99 / 100.000$ em 1970 para 278,61/100.000 em 1980 (Tabelas 2 e 3). Este foi o único grupo em que se observou aumento da mortalidade nos anos estudados, revelando portanto, comportamento totalmente distinto do restante da população, masculina e feminina, cujas taxas apresentaram-se progressivamente menores.

A comparação de algumas causas de óbito, em 1970 e 1980, nos diferentes estratos etários, ajuda a esclarecer tal comportamento (Tabela 4). No grupo de 15 a 24 anos, o risco de morrer por causas externas, entre os homens, tornou-se $28 \%$ maior, passando de $169,15 / 100.000$ para $217,14 / 100.000$. Além disso, a taxa de mortalidade feminina por este grupo de causas diminuiu em cerca de $33 \%$, baixando de 40,24/100.000 para $27,11 / 100.000$, o que tornou a RSM por causas violentas duas vezes maior, em $1980 . \mathrm{Na}$ Fig. 3 procurou-se representar a magnitude deste índice, que não deixa dúvidas a respeito da importância crescente das causas externas no perfil de mortalidade entre os homens jovens. Observa-se ainda na Tabela 4 a redução significativa das taxas de mortalidade por tuberculose e pneumonia, importantes causas de óbito neste grupo, na década de 70 , anterior.

Também no grupo etátio de 25 a 44 anos, as causas externas constituíram o único conjunto cuja RSM aumentou, de maneira significativa,

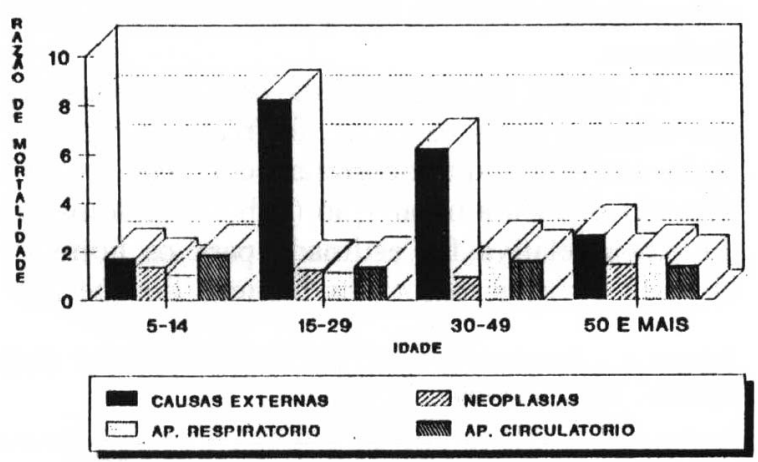

Figura 3. Razão de sobremortalidade masculina por idade $\theta$ causas. Rio de Janeiro, 1980.

de 5,48 para 7,34, entre 1970 e 1980 (Tabela 5). Isto ocorreu às custas do aumento da taxa masculina e também da queda da taxa feminina. Em relação às outras causas de óbito, houve redução importante das taxas de mortalidade em homens e mulheres, principalmente por tuberculose e pneumonia, o que manteve as respectivas razões praticamente inalteradas. 
Tabela 4 - Taxas e diferencial de mortalidade por sexo e causas selecionadas, no grupo de 15.24 anos. Rio de Janeiro, 1970 e 1980

\begin{tabular}{lrrrrrr} 
& \multicolumn{2}{c}{1970} & \multicolumn{3}{c}{1980} \\
Taxas/100.000 & \multicolumn{3}{c}{ Taxas/100.000 } \\
Tipo de Óbito* & Homens & Mulheres & Razão & Homens & Mulheres & Razão \\
\hline Turbeculose & 9,34 & 10,62 & 0,88 & 1,76 & 2,45 & 0,72 \\
Pneumonia & 7,07 & 7,23 & 0,98 & 2,94 & 3,20 & 0,92 \\
Causas Externas & 169,15 & 40,24 & 4,20 & 217,24 & 27,11 & 8,01 \\
\hline
\end{tabular}

* Para a CID de cada causa, ver Metodologia

Tabela 5 - Taxas e diferencial de mortalidade por sexo e causas selecionadas, no grupo de 25.44 anos. Rio de Janeiro, 1970 e 1980

\begin{tabular}{lrrrrrr} 
& \multicolumn{2}{c}{1970} & \multicolumn{2}{c}{1980} \\
Taxas/100.000 & \multicolumn{3}{c}{ Taxas/100.000 } \\
Tipo de Óbito* & Homens & Mulheres & Razăo & Homens & Mulheres & Razão \\
\hline Turbeculose & 57,74 & 24,68 & 2,34 & 13,66 & 5,15 & 2,65 \\
Doenças do Coração & 93,03 & 56,45 & 1,65 & 61,05 & 35,57 & 1,72 \\
Doenças Cérebro. & 31,57 & 26,37 & 1,20 & 26,90 & 24,89 & 1,08 \\
vascular & & & & & & \\
Pneumonia & 24,31 & 9,56 & 2,54 & 13,52 & 6,28 & 2,15 \\
Cirrose & 28,03 & 7,56 & 3,71 & 19,65 & 4,27 & 4,60 \\
Causas Externas & 218,14 & 39,79 & 5,48 & 226,91 & 30,92 & 7,34 \\
\hline
\end{tabular}

- Para a CID de cada causa, ver Metodologia

No grupo mais idoso, entre 45 e 64 anos, observou-se alteração de maior monta somente na RSM por tuberculose (Tabela 6), já que as taxas de mortalidade pelas causas selecionadas apresentaram redução semelhante em homens e mulheres.

A análise mais detalhada do diferencial de mortalidade, entre os sexos, foi realizada em relação aos óbitos que ocorreram em 1980, considerando-se toda a população (Tabela 7). A maior diferença relativa foi estimada para os homicidios, 14,2 vezes maior nos homens do que nas mulheres. Sendo uma das principais causas de óbito masculino, entre 15 e 24 anos, esta foi, possivelmente, a condição que mais contribuiu para o perfil diferenciado desse grupo, apontado anteriormente.

Observa-se ainda, na Tabela 7 , que a mortalidade masculina foi maior do que a feminina em todas as causas estudadas, exceto no caso do diabetes. As causas de óbito cuja RSM foi pelo menos o dobro da feminina foram: homicídios; tumores do aparelho respiratório; causas externas; cirrose hepática; acidentes de transporte; o

Tabela 6 - Taxas e diferencial de mortalidade por sexo e causas selecionadas, no grupo de 45.64 anos. Rio de Janeiro, 1970 e 1980

1970

Taxas/100.000

Tipo de Óbito*

Homens

Mulheres

Turberculose

Neoplasia Maligna

Doenças do Coraçāo

Doenças

Cerebrovascular

Pneumonia

Cirrose

Causas Externas

$\begin{array}{rrr}117,11 & 29,36 & 4,00 \\ 365,76 & 267,61 & 1,37 \\ 616,34 & 280,19 & 2,20 \\ 256,03 & 204,41 & 1,25 \\ & & \\ 51,34 & 25,73 & 2,00 \\ 82,46 & 17,62 & 4,68 \\ 219,78 & 51,73 & 4,25\end{array}$

1980

Taxas $/ 100.000$

\begin{tabular}{rrr} 
Homens & Mulheres & Razão \\
\hline 53,15 & 9,57 & 5,55 \\
321,59 & 215,53 & 1,49 \\
500,39 & 231,97 & 2,16 \\
228,28 & 147,08 & 1,55 \\
& & \\
34,78 & 13,11 & 2,65 \\
67,36 & 12,69 & 5,31 \\
178,31 & 38,70 & 4,61
\end{tabular}

\footnotetext{
- Para a CIO de cada causa, ver Metodologia
} 
Tabela 7 - Diferencial relativo e absoluto entre taxas de mortalidade específicas por sexo e causas selecionadas. Rio de Janeiro, 1980

\begin{tabular}{|c|c|c|c|c|c|}
\hline \multirow[b]{2}{*}{ Tipo de óbito } & \multicolumn{2}{|c|}{ Taxas/100.000 } & \multirow{2}{*}{$\begin{array}{r}\text { Razão } \\
H / M \\
\end{array}$} & \multirow{2}{*}{$\begin{array}{r}\text { Diferença } \\
\mathrm{H} / \mathrm{M} \\
\end{array}$} & \multirow[b]{2}{*}{$\%$ ** } \\
\hline & $\mathrm{H}^{*}$ & $M^{*}$ & & & \\
\hline Doenças do Coração & 269,60 & 171,32 & 1,57 & 98,28 & 23,73 \\
\hline Doenças Isquêmicas & 175,31 & 96,58 & 1,82 & 78,73 & 19,01 \\
\hline Infarto Agudo Miocárdio & 134,05 & 63,42 & 2,11 & 70,63 & 17,06 \\
\hline Doença Cerebrovascular & 134,49 & $\$ 15,06$ & 1,17 & 19,44 & 4,69 \\
\hline Neoplasias Malignas & 152,39 & 105,16 & 1,45 & 47,24 & 11,41 \\
\hline Tumores Ap. Respiratório & 31,47 & 6,77 & 4,65 & 24,70 & 5,96 \\
\hline Pneumonia & 37,60 & 28,49 & 1,32 & 9,11 & 2,20 \\
\hline Asma, Bronquite, Enfisema & 22,68 & 9,18 & 2,47 & 13,50 & 3,26 \\
\hline Diabetes & 30,67 & 35,19 & 0,87 & $\cdot 4,52$ & $-1,09$ \\
\hline Cirrose & 26,39 & 6,13 & 4,30 & 20,25 & 4,89 \\
\hline Causas Externas & 170,28 & 36,96 & 4,61 & 133,32 & 32,20 \\
\hline Acidentes Transporte & 38,86 & 10,43 & 3,73 & 28,43 & 6,87 \\
\hline Homicídio & 60,94 & 4,29 & 14,20 & 56,65 & 13,68 \\
\hline Suicídio & 3,40 & 2,07 & 1,64 & 1,33 & 0,32 \\
\hline D.Infecciosas & 47,65 & 29,13 & 1,64 & 18,52 & 4,47 \\
\hline Outras Causas & 220,72 & 161,76 & 1,36 & 58,95 & 14,24 \\
\hline Todas as Causas & $1.112,47$ & 698,39 & 1,59 & 414,08 & $\ldots$ \\
\hline
\end{tabular}

H. Homens M. Mulheres

- Padronizada pela população feminina do Rio de Janeiro de 1980

** Proporção calculada sobre a diferença absoluta total $(414,08)$

... A soma é maior do que $100 \%$ já que a participação de alguns subgrupos (infarto Agudo do Miocárdio, Tumores do Aparelho Respiratório, Acidentes, Homicidios e Suicidios) é apresentada nas respectivas categorias e também isoladamente.

conjunto representado por bronquites, enfisema e asma; e infarto agudo do miocárdio, em ordem decrescente.

Ainda na Tabela 7, as diferenças absolutas entre as taxas de ambos os sexos foram também avaliadas, já que sua estimativa é importante para a medida da contribuição de cada causa específica à diferença total entre a mortalidade de homens e mulheres $(414,08)$. Os óbitos por causas externas, doenças do coração e neoplasias malignas, considerados em conjunto, corresponderam a cerca de $70 \%$ do diferencial de mortalidade entre homens e mulheres, em 1980. As causas externas foram responsáveis pela maior proporção - 32\% - enquanto as doenças do coração e neoplasias malignas por $24 \%$ e $11 \%$, respectivamente.

Apesar da mortalidade masculina por homicídios ter sido 14 vezes maior do que a feminina, sua participação no diferencial total foi de $13,7 \%$. O infarto agudo do miocárdio, por outro lado, cuja RSM foi de apenas 2,11 , contribuiu com $17 \%$ do diferencial total. Isto se explica pela importância do infarto como causa de óbito na população, apresentando, em conseqüência, maiores taxas de mortalidade do que os homicídios, por exemplo (vide Tabela 7).

\section{Discussão}

$O$ excesso de mortalidade entre as mulheres em relação aos homens é típico de situaçōes de elevada mortalidade, tendo ocorrido no Estado de São Paulo, na década de 40 (Ortiz e Yazaki'1, 1984), e ainda hoje em vários países não industrializados (Waldron ${ }^{27}, 1976$ ). Assim, com a diminuição das taxas gerais de mortalidade, na primeira metade deste século, o fenômeno inverteu-se, passando a ocorrer excesso de mortalidade masculina, na maioria dos países ocidentais (Enterline ${ }^{4}$, 1961). Nos EUA, em 1920, as taxas de mortalidade eram, no máximo, 30\% maiores nos homens do que nas mulheres. Por volta de 1970, as taxas masculinas excediam as femininas em $180 \%$, no grupo de 15 a 24 anos, e em $110 \%$ entre 55 e 64 anos. Na população americana estes são os grupos etários em que a RSM vem apresentando maior incremento (Waldron ${ }^{27}$, 1976).

As causas de óbito apontadas como responsáveis por tal incremento foram os acidentes de trânsito nos jovens, e doenças cardiovasculares, renais e câncer de pulmão, entre os grupos mais idosos. Além disso, a diminuição da mortalidade materna e por câncer de útero contribuíram para o aumento da RSM, nos EUA. 
Também no Brasil, no Estado de Sāo Paulo, a RSM aumentou, especialmente entre os $15 \mathrm{e}$ 39 anos, no período de 1940 a 1980 (Ortiz e Yazakj $^{21}$, 1984). De maneira bastante semelhante à situação descrita para o Município do Rio de Janeiro, os maiores diferenciais entre a mortalidade de homens e mulheres se deslocaram dos grupos etários mais velhos para os mais jovens, à medida que diminuiu a mortalidade geral, em ambos os sexos.

$O$ aumento da sobremortalidade masculina entre os jovens foi causado, em grande parte, pela participação crescente das causas externas em seu perfil de mortalidade, especialmente dos homicídios e acidentes de trânsito, tanto nos EUA quanto no Estado de São Paulo e Município do Rio de Janeiro (Enterline ${ }^{4}, 1961$; Ortiz e Yazaki ${ }^{21}$, 1984). No entanto, a semelhança entre a realidade nacional e a situação americana é bastante restrita. A magnitude da sobremortalidade masculina por homicídio, estimada para as duas regiões do país, não encontra paralelo em nenhum estudo disponivel, relativo a países do Primeiro Mundo. No Estado de São Paulo, em 1980, a mortalidade masculina por homicídio foi 11 vezes maior do que a feminina, entre 15 a 24 anos, enquanto no Rio de Janeiro foi 14 vezes maior, considerando-se todos os grupos etários. Apesar de não ser possível a compararação direta entre o valor das RSM, por nao se ter utilizado a mesma população padrāo dos estudos estrangeiros, é importante citar que, na população americana, em 1980, a RSM relativa aos homicídios foi também a de maior magnitude entre as causas de óbito estudadas, situando-se, porém, em 3,86 .

Os resultados do presente estudo apontam que, no Rio de Janeiro, em 1980, houve predominância de óbitos masculinos relacionados à violência, arteriosclerose, fumo e álcool, que informam sobre as condiçōes de vida da população. Sabemos que estas condiçōes não estão uniformemente distribuídas, e que portanto existem subgrupos de homens jovens especialmente atingidos por este quadro alarmante, de verdadeira guerra civil. Excessos de mortalidade masculina desta ordem de grandeza, de fato, só costumam ser encontrados em situaçōes de guerra, como aconteceu durante os dois conflitos mundiais.

Em situações de paz, a mortalidade do grupo etário entre 15 e 29 anos parece ter se tornado um indicador das condições de vida da sociedade mais sensível do que outros, tradicionalmente usados, como a mortalidade infantil (Sawyer ${ }^{23}$, 1987). Naquele grupo, o saber da ciência médica e, mesmo as intervençōes isoladas de saúde pública, não são suficientes para contrabalançar a deterioração social.

Se, por um lado, as diferenças biológicas entre homens e mulheres podem justificar, em parte, o diferencial de mortalidade nos grupos etários mais idosos, em relação à mortalidade por doenças cardiovasculares e diabetes, e também na mortalidade perinatal e infantil, fica evidente, por outro lado, que as principais causas de óbito em jovens não se explicam através daquelas diferenças. Neste caso, os excessos de mortalidade devem-se aos riscos de vida (ou de morte?) a que é submetida a população masculina.

Gostaríamos de lembrar, por outro lado, que os riscos específicos da população feminina não devem ser subestimados. O uso indiscriminado de anticoncepcionais orais, na vigência de contra indicações formais, com seus efeitos comprovados na ocorrência de complicaçōes tromboembólicas (Rosenberg ${ }^{22}, 1980$, Layde e Beral ${ }^{15}$, 1981) constitui um tipo de "violência", já hoje evitada em outros países. A mortalidade materna, por outro lado, situa-se em patamares muito superiores aos dos países desenvolvidos, constituindo uma "tragédia evitável" (Silva $\left.{ }^{24}, 1991\right)$. E preciso não esquecer ainda que, apesar das menores taxas de mortalidade, alguns trabalhos indicam que a morbidade feminina é maior do que a masculina (Wingard ${ }^{30}$, 1984), indicando a presença ou o "sentimento" mais intenso de doença, ao longo da vida.

Os contrastes dramáticos na mortalidade entre sexos obrigam a constatar os efeitos perversos da organização social vigente no país, que os vem gerando. No campo específico das açōes de saúde pública, só resta fazer algumas indagações. A prevenção da mortalidade masculina precoce é passivel de alguma intervenção específica do assim chamado setor saúde? Se é, certamente não seria o caso de defender a criação de mais um programa voltado unicamente para esta questão. Se não é, há que se conviver com a impotência, culpando os "fatores sociais", em geral, por esta situaçāo e atribuindo à polícia a responsabilidade de diminuir a taxa de mortalidade masculina precoce.

CHOR, D. et. al. [The sex differencial in mortality a city of Southeastern Brazil - 1960, 1970, 1980]

Rev. Saúde públ., S. Paulo, 26: 246 -55, 1992.

Male and female mortality rates in the city of Rio de Janeiro in 1960,1970 and 1980 are studied with a view to analysing the different risks to which men and women are subject by age group.

Mortality differentials by sex and cause were studied by means of male/female mortality ratios, 
relative and absolute differences among rates, and standardized rates. An analysis of differentials by selected groups of cause for 1980 was undertaken. Male mortality rates were higher than the female rates in all age groups in the three years studied, with an increase of the male/female mortality ratio for the 15-34 age group over this period. The excess of male death was mainly due to the increase of deaths from violent causes among young men, a dramatic circunstance that can hardly be changed by "technical" procedures. As regards deaths from other causes, biological determinants and the different risks for men and women should be considered in order better to understand this situation.

Keywords: Differential mortality. Sex factors. Mortality rate. Risk analysis.

\section{Referências Bibliográficas}

1. BANCO MUNDIAL Relatório sobre o desenvolvimento mundial 1990: a pobreza. Rio de Janeiro, Fundação Getúlio Vargas, 1990.

2. BASU, A. M. Is discrimination in food really necessary for explaining sex differentials in childhood mortality? Popul. Stud., 43: 193-210, 1989.

3. CASTRO, M. G. A dinâmica entre classe e genêro na América Latina: apontamentos para uma teoria regional sobre gênero. In: Instituto Brasileiro de Administração Municipal. Rio de Janeiro. Mulher $e$ políticas públicas. Rio de Janeiro, 1991. p. 39-69.

4. ENTERLINE, P. E. Causes of death responsible for recent increases in sex mortality differentials in the United States. Milbank Mem. Fund. Quart., 39: 312-28, 1961.

5. FERREIRA, C.E.C. \& FLORES, L.P.O. As dimensões da mortalidade infantil em São Paulo. Rev. bras. Est. pop., 4: 107-36, 1987.

6. FLEISS, J.L. statistical methods for rates and proportions. New York, John Wiley \& Sons, 1981.

7. FUNDAÇẢO IBGE Censo demográfico de 1960: Guanabara. Guanabara, 1967, v. 1, t. 12, pt.1 (7 Recenseamento Geral do Brasil, 1960).

8. FUNDAÇĀO IBGE. Censo demográfico de 1970: Guanabara. Guanabara, 1973. v. 1, t. 17. (88 Recenseamento Geral do Brasil, 1970).

9. FUNDAÇĀO IBGE Censo demográfico de 1980 , dados distritais: Rio de Janeiro, 1983. v. 1, t. 3, n. 16. (98 Recenseamento Geral do Brasil, 1980).

10. FUNDAÇÃo IBGE. Indicadores sociais: tabelas selecionadas. Rio de Janeiro, 1984. v. 2.

11. FUNDAÇÃO INSTITUTO DE DESENVOLVIMENTO ECONÓMICO E SOCIAL DO RIO DE JANEIRO. Estado do Rio de Janeiro: estatisticas do sistema de saúde, 1977. Rio de Janeiro, FIDERJDIGES|SIPE, 1977.
12. GOVE, W. R \& HUGHES, M. Possible causes of the apparent sex differences in physical health: an empirical investigation. Amer. Sociol. Rev, 44: 126-46, 1979.

13. JOHNSON, A. Sex differentials in comonary heart disease: the explanatory role of primary risk factors. J. Hith soc. Behav., 18: 46-54, 1977.

14. KAHN, H. A. \& SEMPOS, C. T. Statistical methods in epidemiology. Oxford, Oxford University Press, 1989.

15. LAYDE, P. M. \& BERAL, V. Further analysis of mortality in oral contraceptive users. Lancet, 1: 541-6, 1981 .

16. LILIENFELD, A.M \& LILIENFELD, D.E. Foundations of Epidemiology. Oxford, Oxford University Press, 1980.

17. MINISTERIO DA SAÚDE. Estatisticas de mortalidade Brasil, 1980. Brasília, Centro de Documentação do Ministério da Saúde, 1983.

18. MORTARA, G. A mortalidade na América Latina. Rev. bras. Estat. 91/92: 105-35, 1962.

19. ORGANIZAÇÃo PANAMERICANA DA SAÚDE. Manual de classificação estatística internacional de doenças, lesões e causas de b́bito; $\mathbf{8}^{\mathbf{2}}$ revisão. Washington, DC, 1969. (Publicaçāo Cientifica $n^{2}$ 190).

20. ORGANIZAÇĀO MUNDIAL DA SAÚDE. Manual de classificaçäo estatística internacional de doenças, lesões e causas de óbito. 91 revisão, São Paulo, Centro da OMS para Classificação de Doenças em Português, 1978.

21. ORTIZ, L.P. \& YAZAKI, L.M. Aumento do diferencial por sexo da mortalidade no Estado de Sảo Paulo. Rev. bras. Est. pop., 1: 145-70, 1984.

22. ROSENBERG, $L$. et al. Oral contraceptive use in relation to nonfatal myocardial infarction. Amer. J. Epidem., 111: 59-66, 1980.

23. SAWYER, D.O. et al. The impact of urbanization and industrialization on mortality in Brazil. Rapp. trimest. Statis. sanit. mond. 40: 84-95, 1987.

24. SILVA, K.S. Mortalidade matema: avaliação da situação no Rio de Janeiro, no periodo de 1977 a 1987. Cad Saúde públ., 1990 [No prelo].

25. UNICEF. Situação mundial da infância. Brasilia, 1991.

26. VALLIN, J. \& MESLE, F. Les causes de décès en France de 1925 a 1978. Paris, Presses Universiteires de France, 1988. p. 392-9.

27. WALDRON, I. Why do women live longer than men? Soc. Sci. Med, 10: 349-62, 1976.

28. WALDRON, I. Sex differences in human mortality: the role of genetic factors. Soc. Sci. Med, 17: 321-33, 1983.

29. WINGARD, D. L. The sex differential in mortality rates. Amer. J. Epidem., 115: 205-16, 1982.

30. WINDGARD, D.L. The sex differential in morbidity, mortality and lifestyle. Ann. Rev. publ. Hlth, 5: 433-58, 1984

Recebido para publicação em 3/1/1992 Aprovado para publicaçấo em 30/3/1992 